\title{
An Anatomical Study of Mandibular and Accessory Mandibular Foramen in Dry Adult Human Mandibles of South Indian Origin
}

\author{
Padmavathi $\mathrm{G}^{1}$, Suman Tiwari ${ }^{1}$, Varalakshmi KL ${ }^{1}$, Roopashree $\mathrm{R}^{1}$ \\ I' Department of Anatomy, MVJ Medical College and Research Hospital, Bangalore India.)
}

\begin{abstract}
:
Objective: Mandibular foramen is an important anatomical landmark for the inferior alveolar nerve blocks, mandibular osteotomies and implant treatment. A thorough knowledge of the anatomy of the mandibular foramen is very essential hence this study aims to determine the position of the mandibular foramen and incidence of accessory mandibular foramen in south Indian population.

Material and methods: 65 adult human mandibles were obtained from the anatomy department of MVJ medical college. The data was tabulated and statistically analysed.

Results:The mean distance of the mandibular foramen to mandibular notch was 22.1 $3.2 \mathrm{~mm}$. The mandibular foramen to anterior border of ramus distance was $16.9 \pm 2.7 \mathrm{~mm}$, from the mandibular foramen to the posterior border of the ramus was $11.9 \pm 2.2 \mathrm{~mm}$. The mandibular foramen to base of the mandible distance was $24.9 \pm 3.3 \mathrm{~mm}$ and to the gonion was $22.4 \pm 3.2 \mathrm{~mm}$. Accessory mandibular foramen was noted in $41.5 \%$ of the mandibles.

Conclusion: Knowledge of the position of mandibular foramen and incidence of accessory mandibular foramen is useful for the maxillofacial surgeons, oncologists and radiologists in preventing neurovascular complications and misinterpretations.

Indian origin.

Key words: Accessory mandibular foramen, mandibular foramen, morphometry, nerve block, south
\end{abstract}

\section{Introduction}

Mandibular foramen (MF) is situated on the medial surface of the mandibular ramus. It transmits the inferior alveolar nerve (IAN) and vessels into the mandibular canal which curves downwards and forwards into the body of the mandible upto the mental foramen to supply the mandibular teeth. [1]

Mandibular foramen is an important anatomical landmark for surgical procedures like sagittal split osteotomies done to reposition the mandible in prognathism and retrognathia. The main complications encountered during this technique are haemorrhage, injury to the neurovascular bundle, undesired fractures and bone necrosis, hence a thorough knowledge of the mandibular ramus is very essential. [2]

Inferior alveolar nerve block is one of the most common nerve block technique used in dental practise. The success of the procedure mainly depends on placing the needle tip close to the mandibular foramen. Variations in the position of the mandibular foramen and the presence of accessory mandibular foramens are the main reasons accounting for the failure rates of this technique. [3]

The absence of a specific anatomic bony landmark, along with variations in the ramus width and height and the inferior alveolar nerve foramen position, is responsible for failure to achieve profound anaesthesia. Some authors have estimated the failure rate of inferior alveolar nerve blocks to be approximately $20-25 \%$. [4]

A number of nerves like facial, mylohyoid, buccal, transverse cutaneous nerve may pass through the accessory mandibular foramen (AMF) and if local anaesthesia is given in such cases the failure rates are high as the inferior alveolar nerve branches are not completely anaesthetised. [5]

The accessory mandibular foramen can be the site for the spread of tumours following radiotherapy. The knowledge of the additional foramina may be important for the radiotherapists while planning radiation therapy. [6]

Studies have shown racial differences in the anatomy of the mandible. The literature contains conclusive evidence that significant metric, morphological and biological differences are present among the three major racial phenotypes, Caucasoid, Mongoloid \& Negroid. The mandibular and mental foramen are often chosen as reference points because of their stable relation with the base of the mandible, in paleoanthropological studies of the facial skeleton in different populations and for identification of the human remains. $[7,8]$ 
The aim of the study is to determine the precise location of the MF in relation to the borders of the mandibular ramus, to locate the quadrant of the ramus in which the foramen is located in the vertical and horizontal directions and to determine the incidence of occurrence of accessory mandibular foramen in south Indian population.

\section{Material and methods}

Sixty-five adult human mandibles were procured from the department of anatomy, MVJ medical college and research hospital. The mandibles which had sockets for the canine and second molar teeth on the same side were selected for the study \& edentulous and damaged mandibles were excluded. The position of the mandibular foramen from various landmarks were recorded on both the sides of the mandibular ramus. The measurements were taken with the help of the Vernier caliper (Aerospace) calibrated to measure upto $0.1 \mathrm{~mm}$. To precisely locate the mandibular foramen, the following parameters were studied. Fig: 1.

The distance from the inferior border of the mandibular foramen to the base of the mandible (MF-Mb), from the inferior border of the mandibular foramen to the mandibular notch (MF-Mn), from the midpoint of the anterior border of the mandibular foramen to the nearest point on the anterior border of the ramus (MF-Ab) and from the midpoint of the posterior border of the mandibular foramen to the nearest point on the posterior border of the ramus (MF-Pb) were recorded. The mandibular foramen to the gonion (MF-G) was measured on both the sides. The data was collected and subjected to statistical analysis to calculate the standard deviation (SD) and mean.

To locate the quadrant in which the foramen is positioned the following measurements were taken. The mean vertical height was calculated by adding the MF-Mn and MF-Mb distances. The mean horizontal width $(\mathrm{Ab}-\mathrm{Pb})$ was measured from the anterior to posterior borders of the mandibular ramus passing through the middle of the mandibular foramen. The width of the mandibular foramen was calculated by subtracting from ( $\mathrm{Ab}-\mathrm{Pb})$, the sum of the distances of (MF-Ab) and (MF-Pb). The width was divided into halves and this was added to the distance, (MF-Ab) to obtain the mean point of the MF. The percentage of the distance between $\mathrm{Ab}$ - the mean point of the MF with the $(\mathrm{Ab}-\mathrm{Pb})$ distance was calculated to locate MF antero-posteriorly. The vertical localization of the MF was obtained by calculating how much (MF-Mn) represented in the percentage of the addition of the (MF-Mn) and the (MF-Mb) distances. Values from 0 to $25 \%$ comprised the first quadrant; from $26 \%$ to $50 \%$, the second; from $51 \%$ to $75 \%$, the third; and from $76 \%$ to $100 \%$, the forth quadrant.

The centre of the horizontal width of the ramus was measured and the position of the foramen with respect to the centre was analysed.

The mandibles were examined further for the presence of single and multiple accessory mandibular foramen with the help of a magnifying lens and the incidence of its occurrence was calculated. Fig: 2 and Fig: 3.

\section{Results}

The mean distance for each measurement of both sides of the mandible, is tabulated in TABLE:1. The mean distance from the lower border of the mandibular foramen to the mandibular notch on the left and right side were $22.3 \pm 3.4 \mathrm{~mm}$ and $22 \pm 3 \mathrm{~mm}$. The mandibular foramen was positioned from the gonion at a distance of $22.2 \pm 2.9 \mathrm{~mm}$ and $22.6 \pm 3.4 \mathrm{~mm}$ on the left side and on the right side. The shortest distance from the midpoint of the anterior border of the mandibular foramen to the anterior border of the ramus on the left side and right side was $16.9 \pm 2.5 \mathrm{~mm}$ and $16.8 \pm 2.8 \mathrm{~mm}$ and from the midpoint of the posterior border of the foramen to the posterior border of the mandibular ramus was $12.1 \pm 2.4 \mathrm{~mm}$ and $11.7 \pm 2 \mathrm{~mm}$ respectively on the left and right side. The mean vertical distance of the ramus from the mandibular notch to base of the mandible was $47.1 \pm 6.7 \mathrm{~mm}$ and $47 \pm 3.7 \mathrm{~mm}$ on the left and right side. The mean width of the ramus from the anterior to posterior border of the ramus passing through the centre of the mandibular foramen was $32.1 \pm 3.8 \mathrm{~mm}$ and $31.6 \pm 3.7 \mathrm{~mm}$ respectively on the left and right side. The mandibular foramen width averaged $3.1 \pm 2.2 \mathrm{~mm}$ and $3.2 \pm 2.8 \mathrm{~mm}$ on the left and right sides. The mean vertical height of the ramus on the left and right side was $47.1 \pm 6.7 \mathrm{~mm}$ and $47 \pm 3.7 \mathrm{~mm}$ respectively. The foramen was localized to the third quadrant of the mandibular ramus antero-posteriorly, and supero-inferiorly it was localized to the junction between the second and third quadrant. TABLE:2.

The mandibular foramen was situated at an average distance of $0.9 \pm 0.8 \mathrm{~mm}$ posterior to the center of the width of the ramus and at an average distance of $1.4 \pm 0.6 \mathrm{~mm}$ superior to the center of the vertical height of the ramus. 
Unilateral accessory mandibular foramen were noted in $29.2 \%$ and bilaterally AMFs seen in $12.3 \%$ of the cases TABLE:3.

\section{Discussion}

Mandibular foramen is an important landmark for mandibular orthognathic surgeries like vertical ramus osteotomy, inverted L-osteotomy. The IAN is at a greater risk of injury during the surgical procedure. The development of implant techniques increased the interest in the mandible anatomy, specially the mandibular foramen localization.

Daw, J.L, [2] obtained anatomical measurements related to the mandibular foramen from 57 formalin-preserved non-Asian hemi-mandibles, and found that great variability in the position of MF. The author further stresses the importance of being familiar with the relationship of the mandibular foramen, would assist in performing properly a sagittal split of the ramus and reduce the chance for an unfavourable split.

The morphological knowledge of the MF is of paramount importance during the dental procedures of the lower jaw, as structures that go through this foramen should be preserved. The AMF are known to transmit the branches of the inferior alveolar nerve and extra blood vessels which supply the bone. [9] The position of the MF is variable, making it difficult to anaesthetise the IAN successfully. [10]

There are significant differences reported in the location of MF among different ethnic groups. Mbajiorgu EF [11] studied 38 dry mandibles of adult black Zimbabweans and recorded that the mandibular foramen on an average lies at about $2.56 \mathrm{~mm}$ (right) and $2.08 \mathrm{~mm}$ (left) behind the midpoint of rameal width and at approximately $3 \mathrm{~mm}$ superior to the midpoint of rameal height on both sides. The mean anterior and posterior rameal widths were respectively $18.95 \pm 0.41$ and $14.30 \pm 0.35 \mathrm{~mm}$ while the mean rameal heights were respectively $22.50 \pm 0.50$ (upper) and $28.44 \pm 0.65$ (lower). In the present study the mandibular foramen was situated at an average distance of $0.9 \pm 0.8 \mathrm{~mm}$ posterior to the center of the width of the ramus. The mean rameal width anterior and posterior were $16.9 \pm 2.7 \mathrm{~mm}$ and $11.9 \pm$ $2.2 \mathrm{~mm}$. The mean rameal heights were $22.1 \pm 3.2 \mathrm{~mm}$ (upper) and $24.9 \pm 3.3 \mathrm{~mm}$ (lower).

Oguz O, [12] studied 34 dried young Turkish adult human male mandibles and noted the distance of the mandibular foramen to the angle of the anterior ramus were $16.9 \mathrm{~mm}$ on the right and $16.78 \mathrm{~mm}$ on the left. The distance to the posterior side of the ramus was $14.09 \mathrm{~mm}$ on the right, and $14.37 \mathrm{~mm}$ on the left. The narrowest anteroposterior diameters were $32.8 \mathrm{~mm}$ on the right and $32.05 \mathrm{~mm}$ on the left. The mandibular foramen was $0.5 \mathrm{~mm}$ posterior to the centre of the ramus on the right and $0.75 \mathrm{~mm}$ on the left. The distance of the lowest point of mandibular notch to the foramen was $22.37 \mathrm{~mm}$ on the right and $22.17 \mathrm{~mm}$ on the left. The distance from the mandibular foramen to the inferior border of the ramus in the mid position of the ramus was $30.97 \mathrm{~mm}$ on the right and $29.75 \mathrm{~mm}$ on the left side. The present study is comparable to the study of Oguz.O.

104 dried mandibles (65 males, 39 females) from Korean cadavers were studied by Sang Wan Lee. [13] According to him the mandibular foramen was located posteriorly to the midpoint on the Anteroposterior (AP) width of the ramus. It was located at $57.3 \%$ of AP width from the coronoid notch and $56.5 \%$ from the occlusal plane. The mandibular foramen and the tip of lingula were located superiorly to the midpoint on the vertical height of the ramus, on the $48.5 \%$ and $35.7 \%$ of vertical distance from the coronoid notch respectively.

Hayward et al, assessed 107 dried mandibles and demonstrated that the Antero-posterior location of the MF is at the third quadrant and non-significant differences were noted between the studied samples and between the right and left ramus. [14]

The passage of blood vessels and nerves makes the AMF clinically important. The embryological basis of the occurrence of the AMF has been described in the literature. [15] During development, initially, there are three inferior alveolar nerves, which innervate each of the 3 groups of the mandibular teeth. Later, there is a fusion of these nerves and a single inferior alveolar nerve is formed. The incomplete fusion of these 3 nerves leads to the development of double mandibular canals. It was reported that, in $60 \%$ of the cases, the mandibular canal was found to have the entire inferior alveolar nerve passing through it, while, in the remaining $40 \%$ cases, the nerves were found to be scattered.[15] The alternate route through AMF and the awareness about its position is important for achieving successful inferior alveolar nerve blocks. The presence of AMF makes it more vulnerable to perineural spread of tumour cells from cortical to cancellous part of bone.[6] The knowledge of AMF may thus be important for radiotherapist in planning radiation therapy. AMF in the present study was found in $41.5 \%$ of the cases, unilaterality in $29.2 \%$ cases, bilaterally in $12.3 \%$ of the cases.

When the AMF was present behind the main foramen and was followed by a canal directed anteroinferiorly, it was named as Serres canal.[16] In their study, it had an incidence of $42.6 \%$ with $23.4 \%$ unilaterality. The commonest location of AMF in the present study was postero-superior to the main foramen.

\section{Conclusion}


The present study of the morphometry of the mandibular foramen and the incidence of accessory mandibular foramen will provide useful information to the dental, maxillofacial surgeons, radiologist and oncologist performing operations on the mandible to prevent complications, misinterpretations and to plan and develop newer techniques for nerve blocks and mandibular surgeries.

Macroscopic observations revealed the presence of accessory foramina in most of the investigated mandibles. The anatomical variability of incidence and position of accessory foramina should be considered to avoid nerve damage and incomplete nerve blocks in various surgical procedures. These foramina may also be used to give additional local regional anaesthesia. Knowledge of the commonest positions and incidence will be beneficial for oncologists and oro-maxillofacial surgeons in planning graft implants.

\section{References}

[1]. Williams,P.L.,Bannister, L.H.Berry, M.M.,Collins.P.,Dyson.M.,Dussek.J.E.et.al. Gray's anatomy38th.ed.churchill Livingstone, 2000,576-7

[2]. Daw,J.L Jr., de la Paz, M.G., Han.H., Aitken, M.E., Patel,P.K, The mandibular foramen: an anatomic study and its relevance to the sagittal ramus osteotomy, J Craniofac Surg, 10(6),1999, 475-9.

[3]. Erika Cvetko, Bilateral anomalous high position of the mandibular foramen: a case report, Surg Radiol Anat,10,2013.

[4]. Kanan Shah, Pratik Shah, Ajay Parmar, Study of the Location of the Mandibular Foramina in Indian Dry Mandibles, Global Research Analysis, 2(7), 2013,128-30

[5]. Das.S and Suri,R.K, An anatomico-radiological study of an accessory mandibular foramen on the medial mandibular surface, Folia Morphol,63,2004,511-13.

[6]. FunibundaK and Matthews J.N.S, Relationship between accessory foramina and tumour spread in the lateral mandibular surface, J Anat, 195, 1999,185-90.

[7]. Komar,D., Lathrop.S, Frequencies of morphological characteristics in two contemporary forensic collections: implications for identification, J Forensic Sci,51(5),2006,974-8.

[8]. Neiva, R.F., Gapski,R., Wang H.L,Morphometric analysis of implant-related anatomy in Caucasian skulls, J Periodontol,75(8),2004,1061-7

[9]. Jeyaseelan,N., Sharma, J.K, A morphological study of unnamed foramina in the North Indian human mandibles and their possible role in neurovascular transmission, Int J Oral Surg, 13,1984, 239-42.

[10]. Nicholson, M.L, A study of the position of the mandibular foramen, Anat Rec, 212(1), 1985, 110-112

[11]. Mbajiorgu, E.F., A study of the position of the mandibular foramen in adult black Zimbabwean mandibles, Cent Afr J Med, 46(7), 2000, 184-90.

[12]. Oguz,O.1., Bozkir, M.G, Evaluation of location of mandibular and mental foramina in dry, young, adult human male, dentulous mandibles, West Indian Med J, 51(1), 2002, 14-6.

[13]. Sang Wan Lee, et al, A Morphometric Study on the Mandibular Foramen and the Lingula in Korean, Korean J PhysAnthropol, 25(4), 2012, 153-166.

[14]. Hayward, J., Richardson, E.R., Malhotra, S.K, The mandibular foramen: Its anteroposterior position, Oral Surg Oral Med Oral Pathol, 44,1977, 837-843.

[15]. Chavez Lomeli, M.E., MansillaLory J., Pompa, J.A., Kjaer I., The human mandibular canal arises from three separate canals, innervating different tooth groups. J Dent Res,75,1996,1540- 4.

[16]. Galdames, I.C., Matamala, D.A., Smith, R.L. Is the conduct of serres an anatomical variation in adults? ,Int J Morphol, 27, 2009, 43- 7 .

TABLE:1.Parameters to locate the mandibular foramen from various landmarks on the medial surface of the mandibular ramus.

\begin{tabular}{|c|l|l|c|}
\hline Measurements & Side & Mean \pm SD & Total mean \pm SD \\
\hline \multirow{2}{*}{ MF-Ab } & Left & $16.9 \pm 2.5$ & $16.9 \pm 2.7$ \\
\hline \multirow{2}{*}{$\mathrm{MF-Pb}$} & Right & $16.8 \pm 2.8$ & $11.9 \pm 2.2$ \\
\hline $\mathrm{MF}-\mathrm{Mn}$ & Reft & $12.1 \pm 2.4$ & $22.2 \pm 3.2$ \\
\hline \multirow{2}{*}{$\mathrm{MF}-\mathrm{Mb}$} & Left & $22.3 \pm 3.4$ & $24.9 \pm 3.3$ \\
\hline \multirow{2}{*}{ MF-G } & Right & $22.0 \pm 3.0$ & \\
\hline & Left & $24.8 \pm 3.3$ & $22.4 \pm 3.2$ \\
\hline
\end{tabular}


TABLE :2 Metric measurements of the mandibular ramus and mandibular foramen.

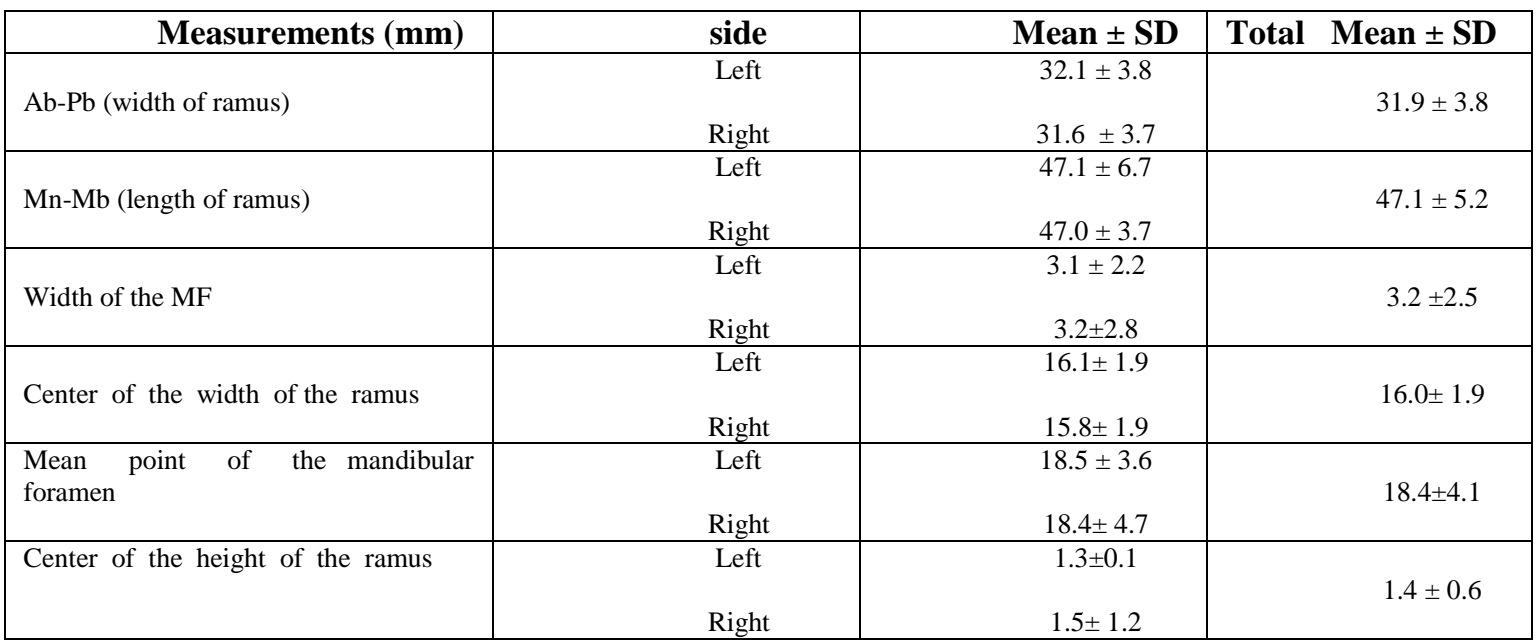

TABLE: 3. Incidence of the presence of AMF in 65 dry adult human mandibles.

\begin{tabular}{|l|c|c|}
\hline AMF & Number & Percentage \\
\hline $\begin{array}{l}\text { Right side } \\
\text { (unilateral) }\end{array}$ & 08 & $12.3 \%$ \\
\hline $\begin{array}{l}\text { Left side } \\
\text { (unilateral) }\end{array}$ & 11 & $16.9 \%$ \\
\hline Bilateral & 08 & $12.3 \%$ \\
\hline Absent & 38 & $58.5 \%$ \\
\hline
\end{tabular}

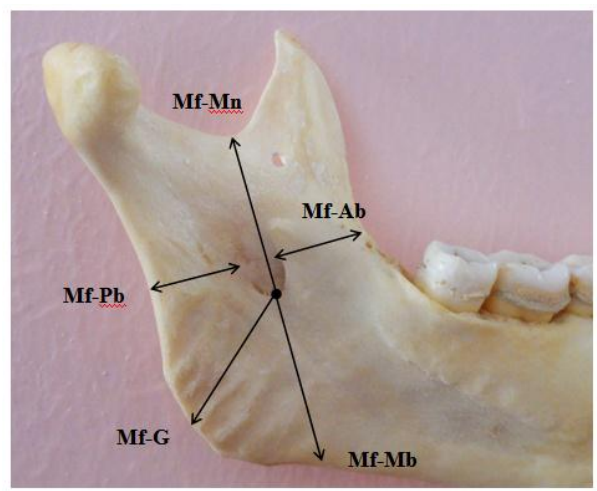

Figure: 1. Figure showing measurements taken from various landmarks on the medial surface of the mandibular ramus to determine the position of the mandibular foramen

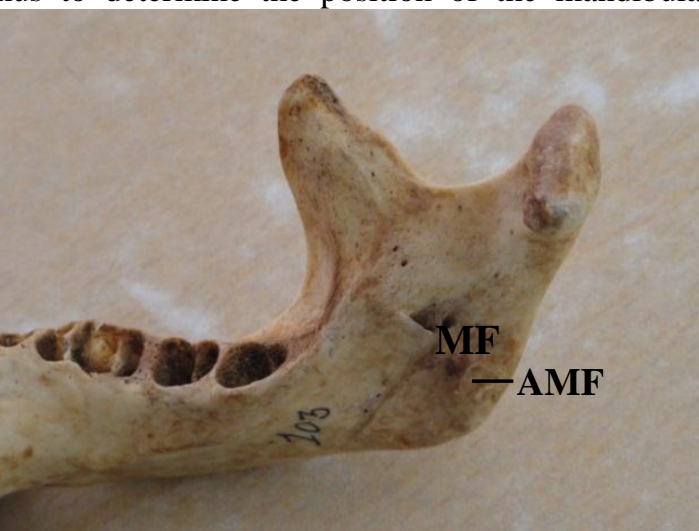

Figure: 2. Medial surface of the mandible showing the presence of mandibular foramen and single AMF. 


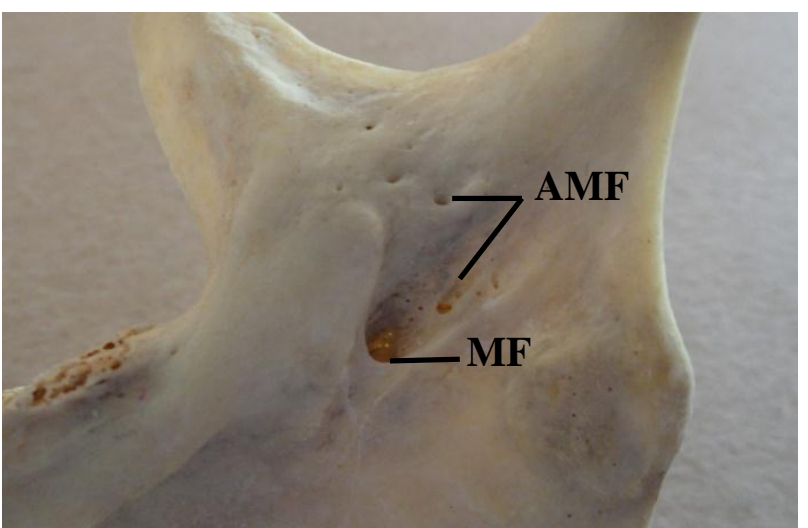

Figure:3. Picture showing mandibular foramen and the presence of multiple accessory mandibular foramen on the medial surface of the mandibular ramus.

Legends for the tables and figures:

MF - Mandibular foramen

AMF- Accessory mandibular foramen

Mf-Mn - Mandibular foramen to mandibular notch

$\mathrm{Mf}-\mathrm{Ab}$ - Mandibular foramen to Anterior border of ramus

$\mathrm{Mf}-\mathrm{Pb}$ - Mandibular foramen to Posterior border of ramus

$\mathrm{Mf}-\mathrm{Mb}$ - Mandibular foramen to the base of the mandible

Mf-G - Mandibular foramen to the Gonion

$\mathrm{Ab}-\mathrm{Pb}$ - Anterior border to posterior border of the ramus

$\mathrm{Mn}-\mathrm{Mb}$ - Mandibular notch to the mandibular base 